\title{
Preface: Unsolved Problems of Magnetospheric Physics
}

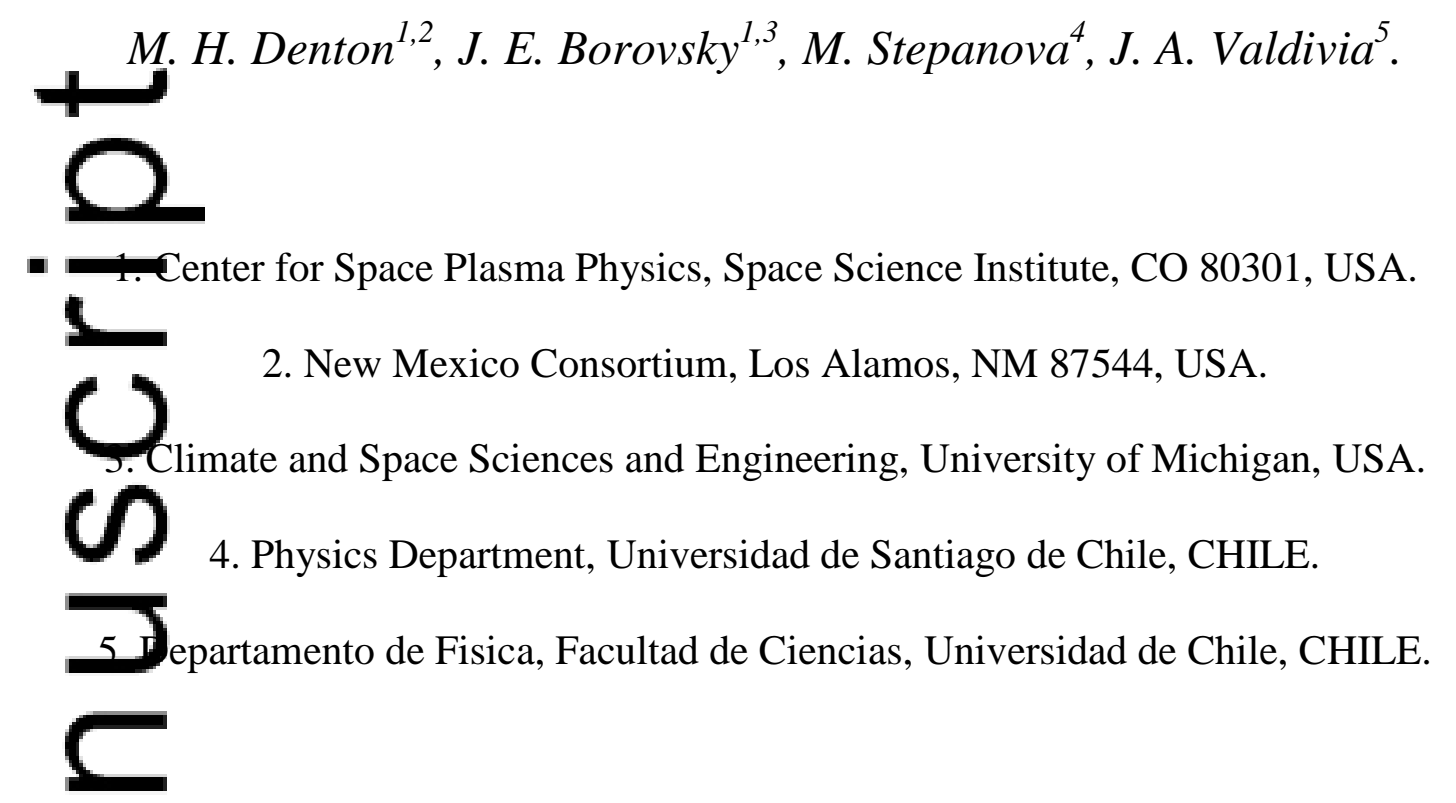

Magnetos 11 physics, as a separate discipline, has existed for little more than 50 years [Stern, 1989; 1996]. Inthat time great strides have been made in understanding both the physical processes at work in the reoin, and also in determining connections and links between these processes. However, for some problems (many of which have been in existence throughout the preceding decades), the community has yet to reach consensus regarding solutions.

To addres this a meeting was convened to bring workers in the field together to discuss how we can define (and hopefully solve) the outstanding problems facing the community. The Unsolved Problems of Magne eric Physics (UPMP) Workshop was held in September 2015 in Scarborough, UK. In contrastan ost other meetings, people were specifically asked not to present and discuss their recent

This is the author manuscript accepted for publication and has undergone full peer review but has not been through the copyediting, typesetting, pagination and proofreading process, which may lead to differences between this version and the Version of Record. Please cite this article as doi: 10.1002/2016JA023362 
results. Rather, they were asked to bring their opinions and thoughts on unsolved problems to the meeting. Short presentations were encouraged after which the audience would debate and discuss definitions of the problems and how they could be overcome. Were new observations required? New missions? Or simply did the community need to work better together to resolve pertinent and outstandin ance questions? Around $50 \%$ of the meeting schedule was devoted to discussion sessions on these topics.

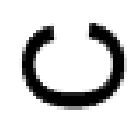

Some outgorys from the meeting have recently been published in a dedicated Special Section of Journal of Geophysical Research on Unsolved Problems of Magnetospheric Physics. Many papers in the Section take the form of a Commentary - a short article discussing specific problems in magnetos physics. Some outstanding questions, and possible routes to their solution, were distilled findience discussions at the meeting:

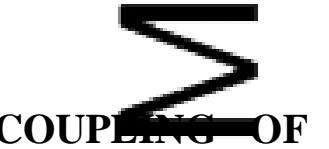

SOLAR WIND AND MAGNETOSPHERE: Although it is integral to understanfing the driving, dynamics, and evolution of the entire magnetosphere, the physics of the coupling betmen solar-wind and magnetosphere remains poorly understood. The solar-wind quantities that control_coupling are not agreed upon and the physical mechanisms underlying the viscous interaction are unknown. The presence of the magnetosheath creates additional difficulties for understanding the coupling.

MAGNETOSPHERIC PLASMAS: There is an almost complete absence of information about 
magnetospheric plasma properties and mass density along the dayside magnetopause. This absence prevents us from assessing the importance of heavy ions and magnetospheric mass density for the mass-loading of dayside reconnection, and the resulting feedback of the magnetosphere on solarwind/magnetosphere coupling (Note: since the workshop the MMS mission has made some progress on addres hase topics).

RADIAT TON BELT MODELING: To improve predictions, the community should aim to transition away fror generic modeling of the radiation belts during averaged conditions, and towards eventspecific modeling. Such a transition would require use of in-situ measurements of the plasma environment (e g. total electron density), the use of event-specific diffusion coefficients, and eventspecific knowledge of the magnetic field configuration.

CHORU GwA VES AND THE RADIATION BELTS: Analysis of time-series data shows that chorus we ing the magnetosphere are intense and bursty, and that the wave-normal angle of chorus can changeby upwards of 50 degrees within a single rising tone. However, all global prediction-based

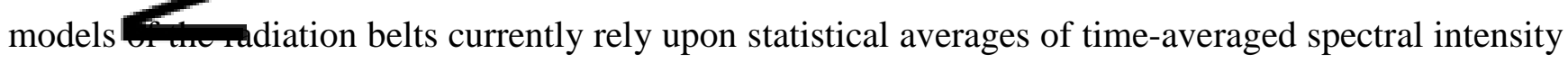
data, which we know to grossly underestimate the instantaneous wave amplitudes. The consequences of using tire-gveraged spectral intensity data when studying chorus waves needs to be assessed, and the importance of large-amplitude oblique whistler-mode waves for radiation belt dynamics needs to be determined.

RADIATLNBELT PRECIPITATION: For energetic particle precipitation we have a theoretical framework that appears to explain the drivers of precipitation, and we have experimental 
measurements, but these are not consistent with one another. This has implications for understanding both the efficacy of radiation belt loss processes, and the impact of those losses on the coupled climate system. In order to address this issue dedicated measurements that are focused on energy-resolved, bounce-Foss-cone, precipitation fluxes, are required.

THE MANTOSPHERE INFLUENCING THE ATMOSPHERE: Research has shown that energetic lectron and ion precipitation, from the magnetosphere into the atmosphere, has implications for the coplea climate system. The chemical changes in the atmosphere produced by the precipitation needs to gtydied in much greater depth than it has been to date. The statistical link between geomagnetic activity and the atmosphere's North Atlantic oscillation has yet to be explained.

LOW-ENERGY ELECTRONS AND IONS: Low-energy electrons and ions ( 0.1-100s eV) permeate magnetosphere although only limited work on understanding and quantifying their general mor hogy and dynamics has been carried out. Precise measurements of the thermal plasma ( 0.1 to a eve $)$ in the magnetosphere are essential to solve a variety of science questions. However, such daratarearrently unavailable due to the inherent technical challenge of routinely measuring this population. This issue needs to be addressed for the community to fully determine the role played by such low-reery particles. Further studies of: (i) the role of low-energy electrons in modulating wavegrowth in the magnetosphere, and (ii) the role of warm plasma cloak ions upon solarwind/magnetosphere coupling, are also required.

PLASMACDLERE REFILLING: Model calculations of plasmaspheric refilling rates do not agree with experimental measurements in the outer plasmasphere. Varying parameters in physics-based 
refilling codes appears insufficient to correct this disagreement. In addition, a robust experimental description of the ion composition of the plasmasphere, as a function of: (i) location, (ii) stage of refilling, and (iii) storm-time conditions, is urgently needed. Analysis of refilling also currently lacks the globalcontext that can be provided by EUV imaging of the plasmasphere.

MAGNE mapping the magnetic field between the ionosphere and magnetosphere. Consequently, our understan (ng) connections between magnetospheric phenomena and ionospheric phenomena remains ambious. For system science, understanding connectivity is crucial. Improved magnetic mapping would increase our ability to use auroral forms in the atmosphere to image and diagnose the processes acting in the magnetosphere. At high latitudes, and/or during active times, this mapping is very diff with implications for magnetic mapping between, and understanding of, auroral streamers and flasma-sheet flow channels.

IONOSFERIC CONDUCTIVITY: Ionospheric conductivity may be one of the least-well quantifierarables in the coupled ionosphere-magnetosphere system. The research community needs a readily gccessible code that can provide quantification of the global Pedersen and Hall conductivity as a function solar wind and IMF values. Such a tool is not presently available. Producing such a tool should he-a community priority.

IONOSPFERIC ION OUTFLOW: It is known that global simulations predict significant changes in 12

the behan the magnetosphere when ionospheric outflows are included. Improved parameterizations of the rates of ion outflow as functions of latitude and local time, geomagnetic 
activity, and solar and solar-wind conditions, are urgently needed for implementation in global simulation codes.

AURORAL ARC GENERATION: The physical mechanisms in the magnetosphere that produce and activate atroral arcs remain unknown. Although auroral arcs are sites of intense energy conversion, is unknoyn. Understanding the emergence and activation of low-latitude auroral arcs would provide vital clues abogt substorm onset and substorm physics. Understanding auroral arc formation is one of the longstanding unsolved issues in the whole of magnetospheric physics.

SUBSTORMS: There remains an urgent need to tie down the timing of substorm evolution and associated auroral features. Much uncertainty exists regarding this issue. In part, this is likely due to the need common definitions of the specific magnetospheric and ionospheric signatures of substorms and pseudo-breakups. Improved magnetic mapping is a further essential ingredient in linking swastorm signatures detected in the magnetosphere with those seen in the ionosphere (see above).

SIMULATIONS: For future generations of global simulations, which are able to model the solarwind-driv 1 magnetosphere (and the development of evolving radiation belts), a methodology to account forion and electron injections by substorms is needed. This would involve either developing MHD codes that can accurately capture substorm physics, or forcing substorm effects in simulations, via tempowmolels of field collapse and induction electric fields. 
It is clear from the list of topics above that our community has many opportunities for a busy future. Understanding of individual processes has, to date, largely followed the tried-and-tested methodology of breaking coupled systems down into individual sub-components, and investigating these subcomponerts in isolation. Such methodology has proven highly successful with regard to magnetos ariphysics over the last 50 years.

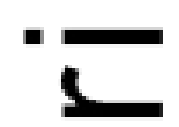

The abov list of "known unknowns" documents our lack of understanding of issues of which the community aware. It is for these issues that we can, at the very least, see a path towards a solution. A challenge-over the next 50 years will be linking together our understanding of individual processes to form a system-wide model of the magnetosphere that is fully-coupled and provides improved predictabi Of even more interest, perhaps, will be the discovery and investigation of "unknown unknown yet-to-be discovered processes at work in the magnetosphere, which will be revealed in the 50 years. These processes will, undoubtedly, challenge our understanding of magnetoprerte physics in ways that we, as yet, cannot grasp.

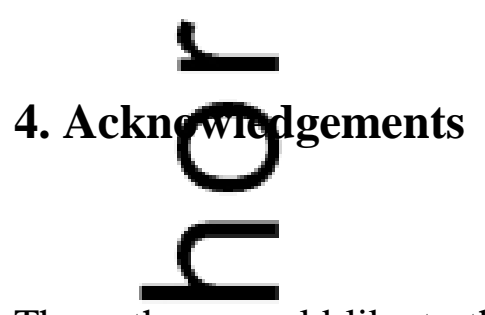

The authors wguld like to thank all who attended the UPMP workshop, and those who worked in the backgrouna, ty make the meeting such a success. Particular thanks are due to Mark Clilverd, Jo Denton, Jame-Denton, Reiner Friedel, Stephen Fuselier, Dennis Gallagher, Larry Kepko, Larry Lyons, 
Mona Kessel, Mike Liemohn, Geoff Reeves, Craig Rodger, Michelle Thomsen, Brian Walsh, Lynn Wilson III, Dan Weimer, and Dan Welling, for their help, useful comments, and suggestions. MHD also wishes to thank Daphne Frank and all staff at The Crown Hotel, Scarborough for their assistance in hosting the workshop. This work was supported at New Mexico Consortium by RBSP-Energetic Particle, acition, and Thermal Plasma funding under NASA's Prime contract no. NAS5-01072, at the Spe Science Institute by NSF Solar-Terrestrial Program grant AGS-1261659 and NASA Heliophy $(5)$ LWS TRT Program grant NNX14AN90G, and Proyecto Basal 041531SSSA NTEXCELE at Universidad de Santiago de Chile.

\section{References}

Stern, D. W. Wrief history of magnetospheric physics before the spaceflight era, Rev. Geophys. 27, $103-114,7989$

Stern, D. R. A brief history of magnetospheric physics during the space age, Rev. Geophys. 34, 1-31, 1996.

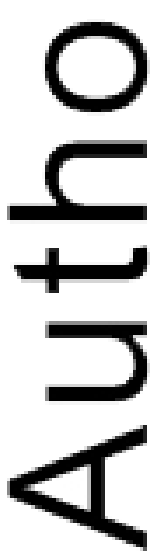

